\title{
A Smartphone Based Method to Enhance Road Pavement Anomaly Detection by Analyzing the Driver Behavior
}

$\begin{array}{ll}\begin{array}{l}\text { Fatjon Seraj } \\ \text { Pervasive Systems } \\ \text { University of Twente, NL. } \\ \text { f.seraj@utwente.nl }\end{array} & \begin{array}{l}\text { Nirvana Meratnia } \\ \text { Pervasive Systems } \\ \text { University of Twente, NL. } \\ \text { n.meratnia@utwente.nl }\end{array} \\ & \\ \text { Kyle Zhang } & \text { Paul J.M. Havinga } \\ \text { Pervasive Systems } & \text { Pervasive Systems } \\ \text { University of Twente, NL. } & \text { University of Twente, NL. } \\ \text { k.zhang@utwente.nl } & \text { p.j.m.havinga@utwente.nl }\end{array}$

\section{Okan Turkes}

Pervasive Systems

University of Twente, NL.

o.turkes@utwente.nl

\begin{abstract}
This paper introduces a method to detect road anomalies by analyzing driver behaviours. The analysis is based on the data and the features extracted from smartphone inertial sensors to calculate the angle of swerving and also based on distinctive states of a driver behaviour event. A novel approach is introduced to deal with the gyroscope drift, reducing the average angle estimation error for curves up to $2^{\circ}$ and the overall average angle error up to $5^{\circ}$. Using a simple machine learning approach and a clustering algorithm, the method can detect $70 \%$ of the swerves and $95 \%$ of the turns on the road.
\end{abstract}

\section{Author Keywords}

Road Monitoring; Anomaly Detection; Smartphone

Sensing; Driver Behaviour;

\section{ACM Classification Keywords}

C.2.4. [Distributed Systems]: Distributed Applications

\section{Introduction}

The coming about of sensor-equipped-smartphones introduced a plethora of possibilities and solutions for ubiquitous sensing. Worldwide population of smartphones has reached 2 billion $^{1}$. The world vehicle population has also increased to an estimated 1

billion $^{2}$. These two factors, give rise to the opportunity of combining the mobility of the vehicles with the 
Table 1: Feature comparison of the related work

\begin{tabular}{|c|c|c|c|c|c|c|c|c|c|c|c|}
\hline \multirow{2}{*}{ Group } & \multicolumn{5}{|c|}{ Sensor } & \multirow{2}{*}{ Smartphone } & \multirow{2}{*}{$\begin{array}{l}\text { Signal } \\
\text { procc. }\end{array}$} & \multirow{2}{*}{$\begin{array}{l}\text { Detection } \\
\text { Classif. }\end{array}$} & \multirow{2}{*}{$\begin{array}{l}\text { Anomaly } \\
\text { Classes }\end{array}$} & \multirow{2}{*}{$\begin{array}{l}\text { Dealing with } \\
\text { Uncertainty }\end{array}$} & \multirow{2}{*}{ Labelling } \\
\hline & acc Hz & gyro Hz & GPS Hz & audio & video & & & & & & \\
\hline [11]PotholePatrol & 380 & no & 1 & & no & No & TD & $\mathrm{ML}$ & 2 & yes & Text \\
\hline [8]Nericell & 310 & no & 1 & yes & no & Yes & TD & Threshold & 1 & no & Text \\
\hline [11]Pertunen et al. & 38 & no & 1 & & no & Yes & FD & SVM & 2 & no & Video \\
\hline [13]Tai et al. & 25 & no & 1 & & no & Yes & FD & SVM & 2 & no & Audio \\
\hline [7]Mednis et al. & 100 & no & 1 & & no & Yes & TD & Threshold & 5 & no & Text \\
\hline [2]Wolverine & 50 & no & 1 & & no & Yes & TD & SVM & 2 & no & Text \\
\hline [12]RoADS & 93 & 100 & 1 & & no & Yes & WD & SVM & 3 & yes & A/V/Text \\
\hline
\end{tabular}

sensors and processing power of the smartphones. Monitoring road conditions in real time help quickly address the problem of road anomalies, reducing accidents and decreasing costs for road maintenance. Road engineers use the Pavement Condition Index to estimate the condition of road pavements. World Road Association in its latest report described in details the importance of an effective road maintenance[1]. Maintenance is four to five times less expensive than the rehabilitation or reconstruction per road kilometer. Road anomalies such as potholes can affect the driving experience and the overall state of the car. Authorities and communities can benefit significantly from the ubiquitous road monitoring, given the financial and economic challenges of our times, as well as the funds shrinkage for road maintenance. Smartphone based road anomaly detection gained momentum some years ago, but still needs to deal with the granularity of the detection (mostly these solutions only detect bumps) and the universality of solutions (to provide more information about the road). In this paper, we describe a solution to detect road anomalies by analyzing driver behaviors using the smartphone sensors. The problem with road anomaly detection systems is the high rate of both false positive (FP) and false negative (FN). The detection rate can substantially be increased if one correlates the detected anomalies with the driver behaviour. Driver behavior on the road is characterized by drivers attitude toward the speed and the turns. Road engineers divide the road curves into two categories: horizontal and vertical curves. Horizontal curves change the direction of the vehicle left or right. The focus of this paper is on horizontal change in direction henceforth called curve. A maneuver on the road is basically a curve or a set of curves. The driver steers the car to follow a curve when he takes a turn, changes the lanes, takes over another car, overtakes a pedestrian or a cyclist, drives on a curvy road or avoids a risky anomaly on the road. Curves can be permanent in the form of road turns or temporal in the form of swerves. Temporal curves are caused by the driver decision to overcome temporal situations on the road, for instance overtaking events or avoiding objects on the road. The main challenges that arise on detecting the road anomalies from the driver behavior information are the classical detection and classification problems that is first detecting all the curves and then finding curves of interest (swerves). To tackle this problem we propose a curve detection and a clustering algorithm and use Stationary Wavelet 
Transformation (SWT) decomposition for signal analysis. To keep computational complexity on the smartphone low, we use the sensor data and precalculated feature set of our previous paper on road anomaly detection system [12]. Given this set of predefined sensors and features, in this paper we present a framework to detect and classify the swerves performed by the driver in an attempt to skip the road anomaly. Based on the geo-location of that curve we propose a clustering algorithm that pinpoints the anomaly and decreases the smartphone GPS error.

\section{Related work}

Several research groups have contributed to the topic of road anomaly detection using inertial sensors [4][8][11][13][2][7]. All these studies made the assumption that the driver will pass through all anomalies. Table. 1 compares the features of these works. Authors of [4],[8], [7] used a time domain accelerometer signal analysis and a threshold approach to detect the road bump and potholes. [4] and [8] used high resolution accelerometers not available in modern smartphones. The other papers presentend in the Table1 use low sampling rate of smartphones' accelerometers. These works considered signal transformation and machine learning techniques for the detection of anomalies. Running these complex algorithms on a smartphone platform involves high power consumption and requires high processing capabilities. None of these studies mentioned the scenario when the driver deliberately skipped the anomalies and how this activity affects the overall results. To the best of our knowledge, only [12] makes use of the gyroscope to detect road anomalies and addresses the problem of driver behavior impact on anomaly detection rate. Only [4] used a clustering algorithm to cluster the anomalies. Driver behavior is mainly studied as a way to detect driver actions behind the steering wheel with the scope to prevent accidents and decrease the accident impact on passengers.

State of the art solutions are based on systems and data received from vehicle sensors. Advanced Driver Assistance Systems utilize driver behavior prediction and a broad range of sensors to assist, complement or substitute the driver according to the traffic situations. Lane Departure Warning system warns the driver intending to leave the lane. Driver behavior is also studied for its impact on traffic load, fuel consumption and resource management. Researches studied the effect of Driving Monitoring Recorders to increase driver awareness and reduce traffic accident[15][14]. Some concluded that there is a correlation between the type of the road and the detected driver behavior[9] . Other works applied Hidden Markov Models algorithms[6][10] on smartphones with augmented signals from the car OnBoard Diagnostics (ODB), to detect and predict the driver behavior from the maneuver states. In [3] the driver behavior is studied to detect critical driving situations on the road while [5] used accelerometer and gyroscope signals with a DTW algorithm to detect the potentially aggressive driving events. They state that using the accelerometer or gyroscope alone is not always possible to distinguish between a left turn and a U-turn.

\section{Methodology}

Our methodology consists of two main steps: swerve detection and clustering

\section{Swerve detection}

The first step to infer road anomalies by analyzing the driver behavior activity, is to detect the road curves. 


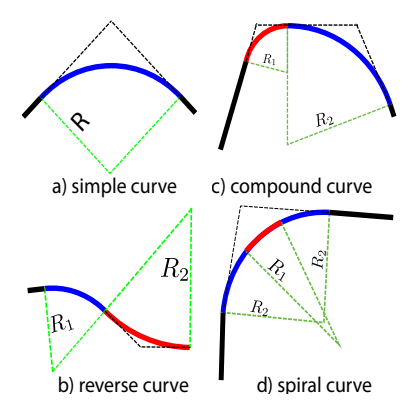

Road engineers divide the road turns into 4 categories, simple, compound, reverse and spiral, as shown in Fig.1. Fig. 3 shows all the features of a curve and forces acting on a car when following that curve, where $a, \nu, R, \theta, C$ and $\omega$ are centripetal acceleration, velocity vector, radius of the curve, angle of the curve,curvature of the curve and angular velocity at $t_{1}$, respectively. From analytical mechanics it is known that: $\omega=\frac{\Delta \theta}{\Delta t}=\theta^{\prime}, \nu=\frac{a}{\omega}, R=\frac{a}{\omega^{2}}, C=\theta R$. Smartphone gyroscope and accelerometer can provide two of the above mentioned parameters. The gyroscope jaw axis, the one parallel to the gravity vector, measures all the angular changes due to the direction changes of the car, and the lateral accelerometer axis measures the centripetal acceleration. Theoretically, the angle of the turn can be calculated by integrating the angular velocity. To calculate the angle of the turn from the gyroscope, the rate of rotation is integrated over time as $\theta(t)=\int_{0}^{t} \frac{\omega}{\Delta t} \approx \sum_{0}^{t} \omega T_{s}$

Figure 2: Left-Right swerve

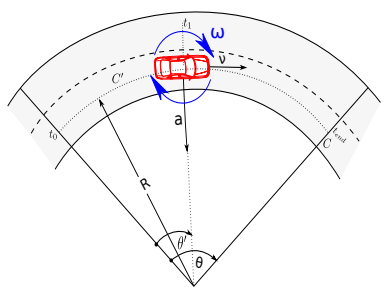

Figure 3: Curve parameters

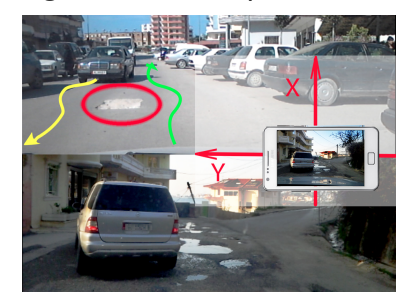

Figure 4: Driver behavior
Unless $\Delta t$ is small enough to capture all the anglular changes, the error of the measurements will be integrated resulting in the gyroscope drift. For certain cases, a simple filter as the moving average, can be enough to reduce the gyroscope drift. Ideally when the car drives on a straight road, the signal of the gyroscope is zero. Whenever the sign of the signal changes from zero, it can be inferred that the car is driving through a curve. When a car drives through a turn like the one in Fig.1a, the signal of the gyroscope will have a sign corresponding to the direction of the curve. If the direction of the turn is the same as the yaw, the sign of the signal will be positive, otherwise it will be negative. However, if the car is driving over a curve like the one in Fig.1b, the sign of the signal will change following the shape of the curve. The transition point corresponds to the precise moment the signal crosses the axis as shown in Fig.2. Knowing the angle of the curve, the slope and the sign of the signal, all curves and their directions can be detected. The road curves sensed by the gyroscope are either road turns (generated by physical turns) or swerves (generated by the driver behaviour). The distinctive feature of these two classes is the angle of the curve. The angle of the swerves cannot be higher than angle of the turn. The angle of the swerve is inversely proportional to the speed of the car, and for a car driving at $20 \mathrm{~km} / \mathrm{h}$ the angle is not bigger than $17.5^{\circ}$

All driver maneuvers can be decomposed into distinctive states. A simple decomposition of swerves of Fig. 2 is:

\section{Detailed States}

- begining left lane change

- crossing left dashed lines

- end left lane change

- passing

- begining right lane chage

- crossing right dashed lines

- end left lane change

A curve varies in angle, radius and complexity. Fig. 5 shows the yaw signal for different type of road curves. Obviously all these signals have distinctive features, in terms of angles, slope, sign, the occourrence order. To increase the accuracy of road anomaly detection, the swerve should happen at the same location and confirmed by at least one detected road anomaly.

The car parking event consists of a set of turns preceded or followed by a stationary state. By decomposing the states of the parking manoeuvre, it is possible to distinguish between parking in a parking lot 


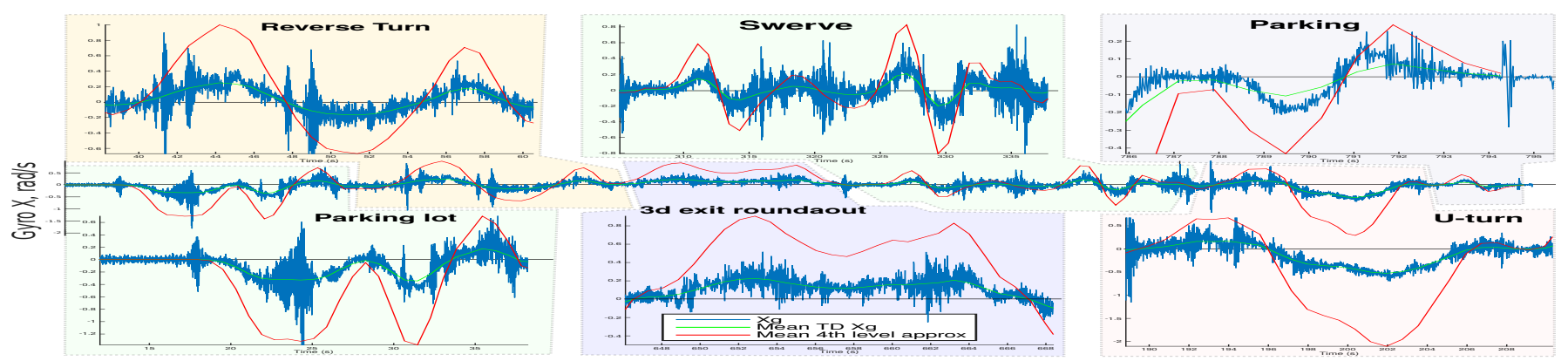

Figure 5: Labelled $x$-axis gyroscope signal for a curve and a swerve

and parallel parking. As shown in Fig.5 parallel parking is composed of small angle turns in opposite directions while parking lot parking consists of a reverse turn on the left, followed by a right turn. The direction of the velocity vector, calculated from the centripetal acceleration and angular velocity $\nu=\frac{a}{\omega}$ shows whether the direction of the maneuver is in reverse. The stopping state of the vehicle, is detected by analyzing the variance feature of gyroscope and accelerometer.

\section{Calculating the angle}

Integration of the angular velocity requires a filtered signal to yield an acceptable result. To this end we use the wavelet decomposition and the precalculated mean feature of the raw gyroscope signal. The mean feature represents the mean for each window of the framed signal. The wavelet denoising method and the moving average can be applied to filter the signal. The wavelet denoising and reconstruction are computationally complexity. The mean of the two consecutive windows is interpolated for the size of the non overlapping windows, resulting in a moving average filter. We compare the angles calculated by both methods and the results obtained by the mean feature were on par with the wavelet denoising method (with an average difference of $0.024^{\circ}$ ). However, since a minimum threshold $T s_{\min }$ is defined, the integration error and the complexity of the algorithm can be further reduced. The angular velocity is integrated only when the algorithm detects the slope in the mean GyrX. The integration continues up to the peak of the curve. If the calculated angle is less than $\frac{1}{2} T s_{\text {min }}$, the angle is too narrow and the calculation stops. The process of angle integration will restart at the point when another slope is detected or if the signal crosses zero. This decision is derived by the geometry of the swerve. Our algorithm uses the raw signal from the yaw axis of the gyroscope and the mean feature from the 4th level of approximation of SWT. All detected curves with an angle more than $T s_{\min }$ will be considered. The detector uses four filters to detect and classify the curves. $T_{\text {turn }}$ filters out all the curves belonging to the turn class. When narrow angles less than $T s_{\min }$ are detected, they are filtered out. If a banked turn continues for a long distance, it will be detected as a chain of small curves on the same direction less than $T_{\text {turn }} . T_{\text {bank }}$ checks these curve segments and if one of them is more than $T_{b a n k} T$, it is considered as a turn. 
When a swerve happens on a banked turn, the sign of the signal may not change. These swerves are detected by $T_{\text {bank }} S$ that checks when slope fluctuation occur on a banked curve. $T_{\text {win }}$ identifies the small curves whose average angular velocity is less than the ratio between angle of the curve $\theta$ and the duration of the curve $t_{\theta}\left(\frac{\theta}{t_{\theta}}\right)$.

\section{Clustering}

To increase the true positive rate we need to identify that a single anomaly happens $n$ times on a given position. To do so we cluster the anomalies and the swerves together based on their geo-location. Our clustering approach is somehow similar to [4]. First a grid of distances is calculated between all positions.

The first distance parameter is used to establish a cluster of minimal elements that fulfill the distance criteria. The second distance parameter is used to include in the cluster the neighbors of the cluster elements that did not fulfill the first criteria. The rationale behind the second parameter is that swerves are not positioned in a radial form, but rather in a linear form. The drivers swerve before reaching the anomaly. Compared to the traditional clustering techniques like DBSCAN, our cluster algorithm excludes the points that do not meet the criteria set by the second parameter. Once the cluster is established, the centroid of the cluster is calculated. The clusters are collaboratively created from reported detections of different vehicles.

\section{Evaluation}

Our evaluation process has different stages. First we evaluate the swerve detector algorithm on labeled data sets using 3-fold cross-validation. Then we test the algorithm on the unlabeled data sets followed by clustering the results and visualizing them on a map while double checking the results with the video footages. The reported false positives are discarded and the false negatives are labeled and added to the labeled data set. Therefore another round of training and testing session is conducted. The performance of the serve detector is also tested on a scenario regarding aggressive driving. The trip consists of a known start and destination and a very poor quality video footage (night), without GPS data. The path of the trip is reconstructed based on the detected turns and swerves. The angles of the reported turns are compared with a rough angular estimation of the turns obtained from Sketchup application ${ }^{3}$. The mean error of the calculated angle for the turns is $5^{\circ}$ (Fig.7).

Table 2: Total road coverage in $\mathrm{km}$ with different vehicles

\begin{tabular}{|c|c|c|c|c|c|c|}
\hline \multicolumn{2}{|c|}{ Trip Car } & Type & $\mathrm{Km}$ & Area & \multicolumn{2}{|c|}{ Hz Location } \\
\hline 1 & Toyota Corolla & $\mathrm{HB}$ & 22.8 & rur/urb & 54 & $E(N L)$ \\
\hline 2 & Peugeot 306 & $\mathrm{HB}$ & 22.8 & rur/urb & 107 & $E(N L)$ \\
\hline 3 & Skoda Fabia & SM & 16.3 & urban & 54 & $V(A L)$ \\
\hline 4 & Toyota Yaris & SM & 33 & hway/rur & 54 & $V(A L)$ \\
\hline 5 & BMW X3 & SUV & 5.46 & urban & 54 & $V(A L)$ \\
\hline 6 & Citroen C3 & SM & 33 & urban & 130 & $E(N L)$ \\
\hline \multirow[t]{2}{*}{7} & VW Golf & $\mathrm{HB}$ & 5 & urban & 130 & $E(N L)$ \\
\hline & Total / Unique & & 80.6 & & & \\
\hline
\end{tabular}

HB= HatchBack, SM= SuperMini, rur=Rural Road, urb=Urban Road, hway=High Way, E=Enschede, V=Vlora

\section{Experimental setup}

We use Android smartphones, Galaxy S2 and Galaxsy S4 Mini with sensors sampling at $96 \mathrm{~Hz}$ and $63 \mathrm{~Hz}$ respectively in the fastest mode. The smartphones were fixed horizontally on the windshield inside the car, with the back camera facing the road ahead (Fig 4).

The data was collected from different type of roads in and around the city of Vlore in Albania and Enschede in the Netherlands. These roads represent completely different environments, crowded and sloppy roads in Albania versus flat roads with regulated traffic in the 


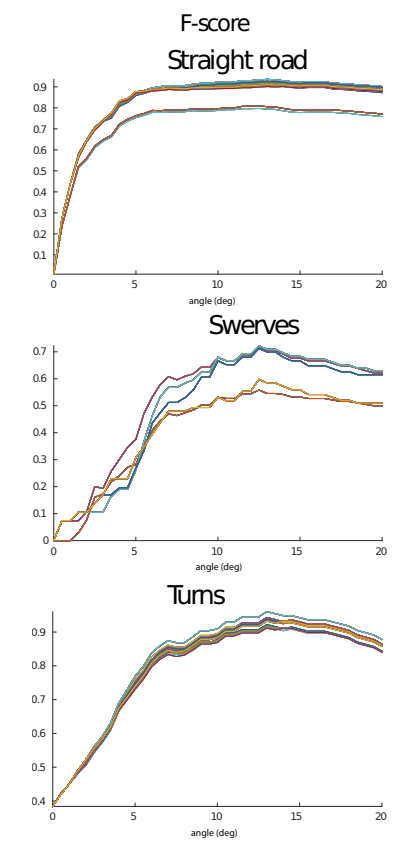

Figure 6: F-score of the detector for $T t_{\text {filt }}=\left\{0^{\circ}, \ldots 20^{\circ}\right\}$ for $T s_{\text {min }}=\left\{0^{\circ}, \ldots 4^{\circ}\right\}, T_{\text {bank }}$ and $T_{\text {win }}$

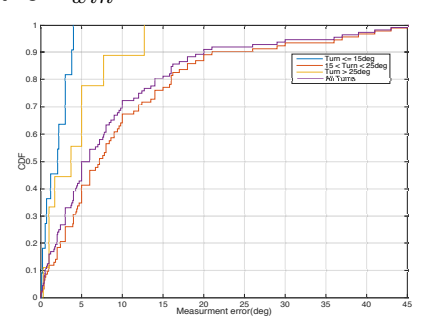

Figure 7: $C D F$ of mean error calculated vs. measured turn angles
Netherlands. Table.2 shows the distances, the environment and the vehicles used to collect the data.

\section{Data preparation}

The ground truth was obtained using the video footages. All noticeable and annotated changes of directions were labeled. The labeling procedure was accomplished using a subtitle editor to annotate all turns and swerves on the road. Signals from all axis of the accelerometer and gyroscope, were windowed in segments of $2^{n}$ samples $<=2.5$ seconds, with $2 / 3$ overlap. More details regarding data preprocessing can be found in [12]. Signal analysis was conducted using SWT at 4 levels of decomposition. The yaw axis of the gyroscope has 5 calculated features for each level of approximation and details. Since swerves and curves are low frequency component signals, we look at the fourth level of approximation representing the $0 \mathrm{~Hz}-3.125 \mathrm{~Hz}$ frequency band of the signal. In Fig. 5 the mean of the approximation for the 4th level of decomposition is smoother than the mean of the raw signal, resulting in less smaller peaks. The short peaks correspond to narrow deviations that are not related to the swerves and curves. The mean of the 4 th level of approximation is used to calculate the slope and the peaks of the signal. Training the classifier consists of finding the best filter parameters fitting every data set available. An exhaustive parameter grid search was conducted simultaneously through all our labeled data. The confusion matrix, accuracy, specificity, sensitivity, recall and f-score were calculated for every iteration. The results were compared and the best parameters were selected analyzing the f-score, precision and recall for the swerve and the turn classes. Fig. 6 shows the calculated f-score through a deep iteration method with increasing threshold values. Once the best parameters are found the algorithm was tested on unlabeled data.

\section{Filter effectiveness}

Our most important filters are $T_{\text {turn }}$ and $T_{\min } . T_{\text {bank }}$ and $T_{\text {win }}$ are mostly auxiliary filters that help reducing the number of FN for the swerve and the turn class. Table.3 shows the confusion matrix before and after $T_{\text {bank }} S$ filter is applied. Evaluation is performed calculating the Positive Predictive Value (PPV) and the True Positive Rate (TPR) . Overall, $T_{b a n k} T$ increased the PPV by $4.1 \%$ for the turn class while $T_{b a n k} S$ increased the TPR of the swerves by $12.7 \%$.

Table 3: Confusion matrix before and after tuning the filters

\begin{tabular}{l|c|c|c|cccc}
\multicolumn{2}{c}{ Straight } & Swerve & Turn & TPR & SPC & PPV & F-score \\
\hline Straight & 223 & 14 & 9 & 0.91 & 0.86 & 0.92 & 0.91 \\
\hline Swerve & 16 & 30 & 1 & 0.64 & 0.95 & 0.64 & 0.64 \\
\hline Turn & 3 & 3 & 85 & 0.93 & 0.97 & 0.89 & 0.91 \\
\hline \multicolumn{7}{c}{ ConfMat after the $T_{\text {bank }} \& T_{\text {win }}$ applied } \\
\hline Straight & 226 & 15 & 5 & 0.92 & 0.90 & 0.94 & 0.93 \\
\hline Swerve & 10 & 36 & 1 & 0.77 & 0.95 & 0.68 & 0.72 \\
\hline Turn & 4 & 2 & 85 & 0.93 & 0.98 & 0.93 & 0.93 \\
\hline
\end{tabular}

\section{Clustering evaluation}

The performance of the clustering algorithm was evaluated by multiple drives on selected tracks at the University Campus. Manholes were swerved around different speeds and angles. Also random swerves were made at random points. The clustering algorithm clustered all emulated anomalies within a certain radius. To find the best cluster parameters that define the radius of the cluster, we took into consideration the error of the GPS, the maximum turning angle and the distance between the peak of the swerve and the point of anomaly. To tune the radius parameters, the same aforementioned grid search algorithm was performed. Fig.9 shows the results of the evaluation phase. Shown 
circled in red are the parking spots. The bike icon are clusters formed from swerves on bike paths intercessions with the road.

\section{Aggressive driving}

An experiment was conducted to detect the aggressive driving behaviour. The aggressive drive style is characterized by the angular velocity on a turn. The turning time of a relaxed drive is more than the turning time for an aggressive drive. The experiment involved a driver driving fast and performing sharp lane changes. By detecting all turns and swerves for this aggressive driving trip, we were able to reconstruct the driving path. The starting point and the destination were known but the GPS signal was not used. The trip was reconstructed using the time between the turns and the angle of the turn. The swerve detector performed better in this situation as the angle of the turns were sharper.

\section{Evaluation results}

Fig.8 shows results of the swerve detector algorithm. The results are manually checked using a map and confirmed with the video footage. Overall the algorithm performed very well on the swerve class, with TPR of $\approx 87 \%$. The turn class was detected with TPR $\approx 97 \%$. PPV for the swerve class was $70 \%$ and for the turn class was $95 \%$. Within the Netherlands, the algorithm performed not so well in distinguishing straight segments from swerves. The dutch roads have often road and bike path intersections. These intersections expand the sides of the road, resulting in a smooth turn. False Discovery Rate (FDR) was 30\% for the swerve class and $5 \%$ for the turn class. This is not a major concern because they are due to individual occurrences and do not influence the clustering.

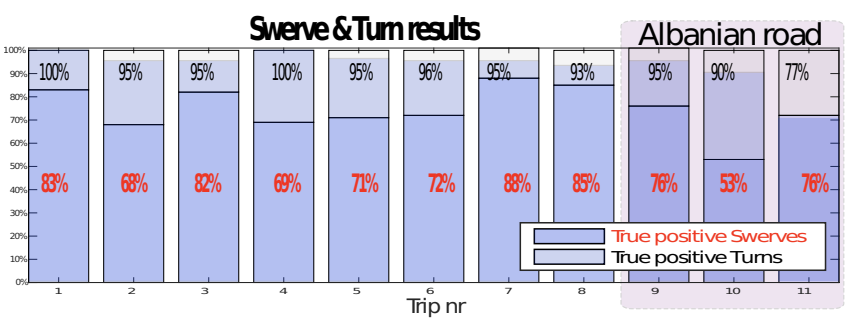

Figure 8: TP and FP of detected swerves and turns

The chaotic traffic in Albania resulted in a great number of swerve events. All swerves related to a road anomaly were detected. The FP were related to the swerves caused by the driver steering wheel calibration or the pavement unevenness. The majority of the swerves were maneuvers due to overtaking. The last trip driven through a highway and a rural road lacks the video footage. The reported FP are the one that we counted on the map. The TP for the turn class was $77 \%$. The reason for this low TP is that on highway the angle of turns is small, whereas the radius is wide and these turns are banked ones. The swerve class in the rural road were falsely detected as turns because this road was very deteriorated and the maneuvers involved turning around those anomalies. From the overall number of the swerve classes, some lane changes were detected as two swerve events. This happened when an overtaking maneuver with lane change left/ long straight/ lane change right format was performed. All detected turns and swerves were clustered together. Overall 120 swerve were detected creating a total of 45 clusters. 15 swerve clusters were due to road pavement anomalies. For the turn class, 277 turns were detected, resulting in 75 turns. All the parking events were clustered together. 


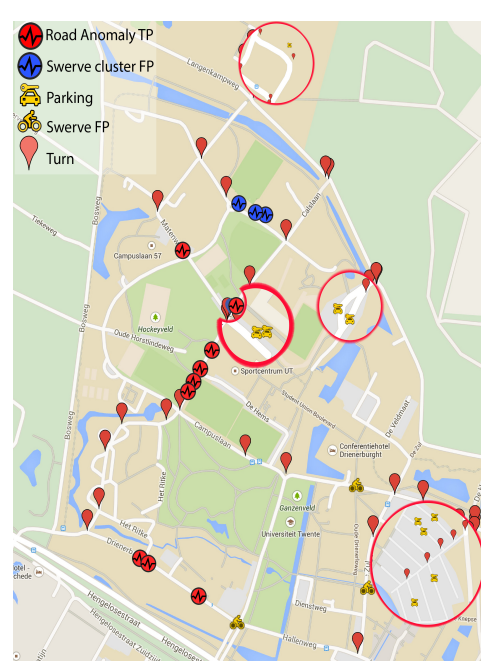

Figure 9: Map of the clustered curves

\section{Conclusions}

In this paper, we described a road anomaly detection method using smartphones. By studying features of the road curves and a simple machine learning technique, we were able to reliably detect swerve and to analyze driver behavior. Overall the TPR of our method for the swerve class is $70 \%$. Apart from swerves, the method can also detect angle of the turns with an average error of $5^{\circ}$ for majority of turns and with average error of $2^{\circ}$ for the turns with an angle less than $15^{\circ}$. Another feature of our method is the ability to detect and distinguish between parking in parking lots and parallel parking. The method performed well on urban environments where speed was limited.

\section{References}

[1] Importance of road maintenance. Executive summary 2014R02EN, World Road Association.

[2] Bhoraskar, R. e. a. Wolverine: Traffic and road condition estimation using smartphone sensors. In Communication Systems and Networks (COMSNETS), 2012 Fourth International Conference on, IEEE (2012), 1-6.

[3] Eren, H. e. a. Estimating driving behavior by a smartphone. In Intelligent Vehicles Symposium (IV), 2012 IEEE, IEEE (2012), 234-239.

[4] Eriksson, J. e. a. The pothole patrol: using a mobile sensor network for road surface monitoring. In Proceedings of the 6th international conference on Mobile systems, applications, and services, ACM (2008), 29-39.

[5] Johnson, D. A. e. a. Driving style recognition using a smartphone as a sensor platform. In Intelligent Transportation Systems (ITSC), 2011 14th International IEEE Conference on, IEEE (2011), 1609-1615.
[6] Kuge, N. e. a. A driver behavior recognition method based on a driver model framework. Tech. rep., 2000.

[7] Mednis, A. e. a. Real time pothole detection using android smartphones with accelerometers. IEEE (June 2011), 1-6.

[8] Mohan, P. e. a. Nericell: rich monitoring of road and traffic conditions using mobile smartphones. In Proceedings of the 6th ACM conference on Embedded network sensor systems, ACM (2008), 323-336.

[9] Paefgen, J. e. a. Driving behavior analysis with smartphones: insights from a controlled field study. In Proceedings of the 11th International Conference on mobile and ubiquitous multimedia, ACM (2012), 36.

[10] Pentland, A. e. a. Modeling and prediction of human behavior. Neural computation 11, 1 (1999), 229-242.

[11] Perttunen, M. e. a. Distributed road surface condition monitoring using mobile phones. In Ubiquitous Intelligence and Computing. 2011 , 64-78.

[12] Seraj, F. e. a. Roads: A road pavement monitoring system for anomaly detection using smart phones (in press).

[13] Tai, Y.-c. e. a. Automatic road anomaly detection using smart mobile device. 1-8.

[14] Ueyama, M. Relationship Between Driving Behavior and Traffic Accidents: Accident Data Recorder and Driving Monitor Recorder. National Highway Traffic Safety Administration, 1998.

[15] Wouters, P. I., and Bos, J. M. Traffic accident reduction by monitoring driver behaviour with in-car data recorders. Accident Analysis \& Prevention 32, 5 (2000), 643-650. 